\title{
Overexpression of microRNA-205 predicts lymph node metastasis and indicates an unfavorable prognosis in endometrial cancer
}

\author{
YONG-JING MA ${ }^{1-3}$, CHUN-FANG HA ${ }^{2,3}$, ZHI-MIAO BAI ${ }^{4}$, HAI-NING LI $^{4}$, YING XIONG ${ }^{2}$ and JIE JIANG ${ }^{1}$ \\ ${ }^{1}$ Department of Obstetrics and Gynecology, Qilu Hospital, Shandong University, Jinan, Shandong 250012; \\ ${ }^{2}$ Department of Gynecology, General Hospital Affiliated to Ningxia Medical University; ${ }^{3}$ Key Laboratory of Fertility \\ Preservation and Maintenance of Ministry of Education; ${ }^{4}$ Ningxia Medical University, Yinchuan, Ningxia 750004, P.R. China
}

Received July 17, 2015; Accepted September 1, 2016

DOI: $10.3892 / 01.2016 .5262$

\begin{abstract}
As an integral component of the surgical staging system, lymphadenectomy for patients with endometrial cancer (EC) remains controversial, particularly in clinical stage I disease that includes not only low-risk, but also high-risk subgroups. In order to maximize the therapeutic effect of lymph node excision for high-risk patients who can potentially obtain survival benefits from it while minimizing its reverse effects in low-risk patients, pre-operative risk stratification of lymph node metastasis is necessary. The upregulation of microRNA-205 (miR-205) in carcinoma of the endometrium has been consistently reported recently and has been found to correlate with poor survival. The current study aimed to investigate whether the overexpression of miR-205 in curettage samples of EC could identify patients who are at a high risk for lymph node metastasis prior to surgery and validate the role of miR-205 as a prognostic marker in EC. Relative quantification detection of miR-205 in curettage and hysterectomy specimens of patients with EC was performed. Prediction of lymph node metastasis based on miR-205 expression, as well as tumor type and grade in curettage samples, was performed for all EC patients and patients with clinical stage I disease. Moreover, survival analysis was conducted. It was observed that miR-205 was significantly and consistently elevated in the curettage and hysterectomy samples of EC relative to normal controls. Furthermore, the overexpression of miR-205 could predict lymph node metastasis with a high accuracy and was revealed again to be associated with a poor prognosis in EC. Prospective and multicentric studies are required to further clarify the value of miR-205 as a promising predictor to stratify risk for lymph node metastasis in EC.
\end{abstract}

Correspondence to: Professor Jie Jiang, Department of Obstetrics and Gynecology, Qilu Hospital, Shandong University, 107 West Wenhua Road, Jinan, Shandong 250012, P.R. China

E-mail: jiangjie2015@qq.com

Key words: microRNA-205, endometrial cancer, lymph node metastasis, prognosis

\section{Introduction}

Endometrial cancer (EC) is the most common malignancy of the female genital system, with a mortality rate only less than that of ovarian cancer in the United States in 2013 (1). In China, there has been a growing tendency for this disease to increasingly occur in younger women $(2,3)$. Although the majority of patients are initially diagnosed at an early stage, $15-20 \%$ of high risk cases are found to suffer from relapse $(4,5)$. The use of lymphadenectomy for EC has remained debatable since the International Federation of Gynecology and Obstetrics (FIGO) (6) proposed surgical staging in 1988. Previously, two randomized controlled trials reported that there was no benefit from systematic lymph node excision in terms of survival in early-stage EC $(7,8)$. In addition, it was shown by a retrospective review that nodal resection was not found to have a survival benefit in the low-risk group, whereas in the medium- and high-risk group, complete staging was correlated with improved 5 -year survival rates (9). It is well known that clinical stage I disease consists of not only low-risk, but also intermediateand high-risk patients. Therefore, lymphadenectomy must be tailored via pre-operative risk assessments to ensure its therapeutic effect, and to avoid unwanted invasiveness and complications such as lymph cyst formation and lower limb lymphedema. Thus far, literature regarding the prediction of lymph node metastasis prior to surgery in EC has been limited and each study has had limitations (10-12). Identification of novel and more reliable molecular markers in pre-operative curettage specimens would be conducive to individualized and more accurate surgical treatment for EC patients.

MicroRNAs (miRNAs/miRs) are small, non-coding RNA sequences containing 19-25 nucleotides, which usually control gene expression by binding to the 3 -untranslated region of their target mRNAs at a post-transcriptional level, either resulting in the degradation of the mRNA transcript or leading to translational repression $(13,14)$. Numerous miRNAs have been revealed to be overexpressed or downregulated in various types of human tumors, thus demonstrating their roles as oncogenes or tumor suppressors (15-18). The altered expression of miRNAs in the majority of tumor types suggests that they can be used as diagnostic or prognostic biomarkers in cancer $(19,20)$. Moreover, the superior stability of miRNAs 
in formalin-fixed paraffin-embedded (FFPE) tissues and their relative stability even in curettage specimens make the clinical utility of miRNAs feasible (21).

It has previously been revealed that miR-205 shows altered expression in a variety of malignancies. In certain cancer types, such as ovarian cancer and nasopharyngeal carcinoma, it serves as an oncogene $(22,23)$, while in prostate cancer, it exerts a tumor suppressive function (24). For EC, however, several studies have consistently achieved results showing that miR-205 is upregulated in EC compared with normal endometrial tissues or plasma samples $(25,26)$.

The goal of the present study was to validate the role of miR-205 as a prognostic marker and to determine its correlation with clinicopathological parameters, including lymph node status, in EC. Notably, the study aimed to investigate whether the overexpression of miR-205 in curettage samples of EC could identify patients who are more likely to develop lymph node metastases prior to surgery, so that a tailored lymphadenectomy could be offered to patients who required it the most and survival benefits could be obtained from the procedure of lymph node dissection.

\section{Materials and methods}

Patients and samples. A total of 360 eligible EC patients admitted to the General Hospital Affiliated to Ningxia Medical University (Yinchuan, Ningxia, China) between January 2006 and December 2010 were enrolled in the current study. All the EC patients were females and the median age was 59 years (range, 35-78). The study was approved by the Ethics Committee of the General Hospital Affiliated to Ningxia Medical University. For the inclusion criteria, all patients had received primary surgical treatment mainly consisting of a hysterectomy, bilateral salpingo-oophorectomy and pelvic lymphadenectomy. Para-aortic lymph node sampling was conducted when suspicious nodes were identified or encountered during the surgery. Patients who were subjected to hormonal, chemical or radiation therapy prior to surgical treatment were ruled out according to the exclusion criteria. The clinical stage was confirmed according to the 1971 FIGO criteria (27). For surgical staging, the 1988 FIGO (28) criteria were applied. Clinicopathological data and follow-up information with regard to survival were retrieved. The date of the last follow-up was December 31, 2014, with a median follow-up time of 55 months (range, 0-96 months). A total of 86 patients succumbed to the EC. The characteristics of the patients are summarized in Table I. The archival FFPE tissue blocks used in this study were collected from the Department of Pathology at the General Hospital Affiliated to Ningxia Medical University. For RNA extraction, tumor samples were obtained from EC patients who underwent curettage and hysterectomy, while normal control endometrial tissues were acquired from patients receiving hysterectomy due to myoma of the uterus at the General Hospital Affiliated to Ningxia Medical University.

RNA extraction. Using archival FFPE tissues, tumor and normal endometrium were identified using the corresponding hematoxylin and eosin-stained sections, the width of which was $2.0 \mathrm{~cm}$. Total RNA was isolated using a miRNeasy FFPE kit (Qiagen, Hilden, Germany) based on the manufacturer's protocols. The quantity of the RNA was assessed using a NanoDrop 1000 spectrophotometer (Thermo Fisher Scientific, Inc., Wilmington, DE, USA).

Reverse transcription and quantitative polymerase chain reaction (PCR). Reverse transcription (cDNA synthesis) was fulfilled by the High Capacity cDNA Synthesis kit (Applied Biosystems, CA, USA) with miRNA specific primers, using $2 \mu 1$ total RNA, performed according to the manufacturer's instructions. The miR-205 and the internal control U6 small nuclear RNA (U6) specific primers was synthesized by Sangon Biotech (Shanghai) Co., Ltd. (Shanghai, China). The primer sequences were as follows: miR-205 forward, 5'-ACACTC CAGCTGGCTCCTTCATTCCACCGGAG-3' and reverse, 5'-TGGTGTCGTGGAGTCG-3'; and U6 forward, 5'-CTC GCTTCGGCAGCACA-3' and reverse, 5'-AACGCTTCA CGAATTTGCGT-3'. Quantitative PCR was performed on a Roche LightCycler ${ }^{\circledR} 480-$ II. All reactions were performed in a final volume of $20 \mu \mathrm{l}$ reaction mixture containing $2 \mu \mathrm{l}$ synthesized cDNA, $2 \mu \mathrm{l}$ of each primer $(100 \mu \mathrm{M}), 10 \mu \mathrm{l} 2 \mathrm{X}$ Roche LightCycler ${ }^{\circledR}$ SYBR Green I Master (Roche Applied Science, Mannheim, Germany), and $6 \mu 1$ water, which was a type of tailor-made solvent of the LightCycler ${ }^{\circledR} 480$ SYBR Green I Master mix. The PCR parameters were a first pre-incubation step at $95^{\circ} \mathrm{C}$ for $5 \mathrm{~min}$, followed by an amplification step with 45 cycles at $95^{\circ} \mathrm{C}$ for $10 \mathrm{sec}, 60^{\circ} \mathrm{C}$ for $5 \mathrm{sec}$ and $72^{\circ} \mathrm{C}$ for $5 \mathrm{sec}$. A melting curve was generated to evaluate the specificity of the PCR products. U6 was used as an internal control to normalize the amount of miR-205. Relative expression level of miR-205 was calculated using the $\Delta \Delta \mathrm{Ct}$ method (29). Each sample was detected in triplicate.

Statistical analyses. All statistical analyses were performed using SPSS program version 17.0 (SPSS, Chicago, IL, USA). The relative expression of miR-205 in different groups was compared by a Mann-Whitney U test. Analyses for associations between miR-205 expression and clinicopathological parameters were made using Pearson's $\chi^{2}$ test. Binary logistic regression was used to evaluate odds ratios (OR) for lymph node metastasis. A receiver operating characteristic (ROC) curve was generated to find a cutoff of miR-205 with optimal diagnostic sensitivity and specificity. Disease-specific survival time was defined as the time from surgery to mortality from endometrial carcinoma. The analysis of disease-specific survival was performed by the Kaplan-Meier method and compared using the Log-rank test. Cox's proportional hazard model was used for multivariate survival analysis and the assessment of prognostic factors. All statistical tests were two-sided and $\mathrm{P}<0.05$ was considered to indicate a statistically significant difference.

\section{Results}

Expression of miR-205 is elevated in curettage and hysterectomy samples of $E C$. It had been previously revealed by certain studies that miR-205 plays a role as an oncogene in EC $(25,26)$. The present study examined the relative expression of miR-205 in curettage and hysterectomy specimens of EC and in corresponding normal endometrial tissues by quantitative PCR. The 
median miR-205 expression level in the endometrial biopsies of EC and in the normal endometrium were 3.5 (range, 1.8-5.0) and 0.2 (range, 0.04-0.37), respectively. The median miR-205 expression level in the hysterectomy samples of EC and in the normal endometrium were 3.5 (range, 1.6-5.4) and 0.2 (range, 0.03-0.40), respectively. The median expression level of miR-205 was upregulated consistently in the curettage and hysterectomy cancer tissues by 17.5-fold when compared with their normal counterparts $(\mathrm{P}<0.0001)$ (Fig. 1).

Correlation between overexpression of miR-205 and clinicopathological parameters in EC. The overexpression of miR-205 was found in 129 (36\%) of the curettage and the hysterectomy specimens. The increased expression of miR-205 in the curettage and hysterectomy samples was significantly correlated with pre-operative histological characteristics, including subtype and grade (both $\mathrm{P}<0.001$; Table II). The upregulated expression of miR-205 in the curettage and hysterectomy samples of EC was also significantly correlated with the presence of lymph node metastasis (both $\mathrm{P}<0.001$; Table II).

The expression of miR-205 in the curettage and hysterectomy specimens was totally concordant. In addition, there were no statistically significant differences between pre-operative and post-operative histological characteristics (subtype and grade), as presented in Table II. The concordance of histological subtype was $94.9 \%$, with 2 patients diagnosed as endometrioid by endometrial biopsy, but validated by final pathological report as non-endometrioid. The concordance of histological grade was $94.0 \%$, with 3 patients diagnosed as grade 1 or 2 prior to surgery and subsequently confirmed as grade 3 by post-operative pathological result.

Overexpression of miR-205 can predict lymph node metastasis for patients with EC. In order to investigate the predictive potential of miR-205 expression for lymph node metastasis, an ROC curve of miR-205 expression was made by comparing patients with and without lymph node metastasis. For all EC patients $(n=360), 46$ patients with lymph node metastasis had been validated by final pathological reports. Lymph node metastasis could be identified with a sensitivity of $50.0 \%$ and a specificity of $85.0 \%$ by pre-operative histological characteristics (Table III). In this study population, the best cut-off of miR-205 expression was a fold-change of 16.0. The expression of miR-205 with a fold-change of $>16.0$ in curettage specimens demonstrated a sensitivity of $71.4 \%$, a specificity of $80.2 \%$, a positive likelihood ratio of 3.55 , a negative likelihood ratio of 0.36 and an accuracy of $79.4 \%$ [area under curve $(\mathrm{AUC})=0.861 ; \mathrm{P}=0.003 ; 95 \% \mathrm{CI}, 0.709-1.000$ ] (Table III; Fig. 2). Based on a combination of pre-operative histological characteristics and miR-205 expression, the predictive ability of lymph node metastasis could be enhanced to a sensitivity of $80.4 \%$, a specificity of $80.3 \%$, a positive likelihood ratio of 4.00 , a negative likelihood ratio of 0.25 and an accuracy of $80.3 \%$ (Table III).

According to the 1971 FIGO clinical staging, the EC patients with clinical stage I and II-IV were 270 (75.0\%) and $90(25.0 \%)$, respectively. On the basis of the final pathological reports, 29 patients with lymph node metastasis had been found among the patients with clinical stage I disease. Lymph
Table I. Clinicopathological characteristics of patients with endometrial cancer.

\begin{tabular}{|c|c|}
\hline Characteristics & $\mathrm{n}(\%)$ \\
\hline \multicolumn{2}{|l|}{ Age, years } \\
\hline$<60$ & $111(30.8)$ \\
\hline$\geq 60$ & $249(69.2)$ \\
\hline \multicolumn{2}{|l|}{ Menopause } \\
\hline No & $107(29.7)$ \\
\hline Yes & $253(70.3)$ \\
\hline \multicolumn{2}{|l|}{ FIGO staging $^{\mathrm{a}}$} \\
\hline I & $267(74.2)$ \\
\hline II & $39(10.8)$ \\
\hline III & $48(13.3)$ \\
\hline IV & $6(1.7)$ \\
\hline \multicolumn{2}{|l|}{ Histological type } \\
\hline Endometrioid & $321(89.2)$ \\
\hline Non-endometrioid & $39(10.8)$ \\
\hline \multicolumn{2}{|l|}{ Histological grade } \\
\hline 1 and 2 & $310(86.1)$ \\
\hline 3 & $50(13.9)$ \\
\hline \multicolumn{2}{|l|}{ Myometrial invasion } \\
\hline No or $<1 / 2$ & $302(83.9)$ \\
\hline$\geq 1 / 2$ & $58(16.1)$ \\
\hline \multicolumn{2}{|c|}{ Lymph node metastasis } \\
\hline Negative & $314(87.2)$ \\
\hline Positive & $46(12.8)$ \\
\hline
\end{tabular}

FIGO, international federation of gynecology and obstetrics. a according to the 1988 FIGO surgical staging system.

node metastasis could be predicted with a sensitivity of $45.0 \%$ and a specificity of $88.0 \%$ by pre-operative characteristics (Table IV). When the best cutoff of miR-205 was evaluated in the endometrial biopsies for predicting lymph node metastasis in this group using a ROC curve, the miR-205 level of 14.5 fold-change was the best, with a sensitivity of $72.5 \%$, a specificity of $84.2 \%$, a positive likelihood ratio of 4.56 , a negative likelihood ratio of 0.32 and an accuracy of $83.0 \%$ (AUC $=0.821 ; \mathrm{P}=0.004 ; 95 \%$ CI, 0.666-0.975) (Table IV; Fig. 3). When combining pre-operative histological characteristics with miR-205 expression, the predictive value of lymph node metastases could be improved to a sensitivity of $82.7 \%$, a specificity of $83.8 \%$, a positive likelihood ratio of 5.19 , a negative likelihood ratio of 0.20 and an accuracy of $83.7 \%$ (Table IV).

Upregulated miR-205 is associated with poor prognosis in patients with EC. It has been reported that the overexpression of miR-205 is negatively correlated with the prognosis of patients with EC (30). To further validate the prognostic significance of miR-205 in EC, Kaplan-Meier survival analysis was performed to assess disease-specific survival. The results revealed that the overexpression of miR-205 was significantly correlated with poor patient survival. As shown in Table V 
Table II. Overexpression of microRNA-205 in curettage and hysterectomy specimens of patients with endometrial cancer associated with clinicopathological variables.

\begin{tabular}{|c|c|c|c|c|}
\hline \multirow[b]{2}{*}{ Variable } & \multicolumn{2}{|c|}{ Curettage specimens } & \multicolumn{2}{|c|}{ Hysterectomy specimens } \\
\hline & $\mathrm{n}(\%)$ & P-value & $\mathrm{n}(\%)$ & P-value \\
\hline Histological subtype & & $<0.001$ & & $<0.001$ \\
\hline Endometrioid & $105 / 323(32.5)$ & & $104 / 321(32.3)$ & \\
\hline Non-endometrioid & 24/37 (64.9) & & 25/39 (64.1) & \\
\hline Histological grade & & $<0.001$ & & $<0.001$ \\
\hline 1 and 2 & $101 / 313(32.3)$ & & 99/310 (31.9) & \\
\hline 3 & 28/47 (59.6) & & $30 / 50(60.0)$ & \\
\hline Lymph node metastasis & & $<0.001$ & & $<0.001$ \\
\hline Absent & $94 / 314(29.9)$ & & $95 / 314(30.2)$ & \\
\hline Present & $35 / 46(76.1)$ & & $34 / 46(73.9)$ & \\
\hline
\end{tabular}

Table III. Prediction of lymph node metastasis according to histological variables and expression of miR-205 (cut-off of 16.0 FC) in curettage specimens for all endometrial cancer patients.

\begin{tabular}{|c|c|c|c|c|c|c|c|c|c|c|c|}
\hline \multirow[b]{2}{*}{ Variable } & \multirow[b]{2}{*}{$\mathrm{n}$} & \multicolumn{3}{|c|}{ Univariate } & \multicolumn{3}{|c|}{ Multivariate } & \multirow[b]{2}{*}{ SNS } & \multirow[b]{2}{*}{ SP } & \multirow[b]{2}{*}{ LR+ } & \multirow[b]{2}{*}{ LR- } \\
\hline & & OR & $95 \% \mathrm{CI}$ & P-value & OR & $95 \% \mathrm{CI}$ & P-value & & & & \\
\hline Curettage histology & & & & 0.002 & & & 0.01 & 0.50 & 0.85 & 3.33 & 0.59 \\
\hline Low-risk & 291 & 1.00 & & & 1.00 & & & & & & \\
\hline High-risk & 69 & 3.01 & $1.77-5.08$ & & 2.76 & $1.22-3.84$ & & & & & \\
\hline miR-205 expression & & & & $<0.001$ & & & 0.02 & 0.71 & 0.80 & 3.55 & 0.36 \\
\hline miR-205<16.0 FC & 266 & 1.00 & & & 1.00 & & & & & & \\
\hline miR-205>16.0 FC & 94 & 3.11 & $1.56-4.63$ & & 2.14 & $1.17-4.15$ & & & & & \\
\hline Curettage histology+ miR-205 & & & & 0.001 & & & 0.01 & 0.80 & 0.80 & 4.00 & 0.25 \\
\hline Low-risk+miR-205<16.0 FC & 261 & 1.00 & & & 1.00 & & & & & & \\
\hline High-risk or/and miR-205>16.0 FC & 99 & 3.06 & $1.10-4.99$ & & 2.55 & $1.18-3.97$ & & & & & \\
\hline
\end{tabular}

Curettage histology low-risk: Endometrioid and grade 1-2; and high-risk: Non-endometrioid or endometrioid grade 3. OR, odds ratio; CI, confidence interval; SNS, sensitivity; SP, specificity; LR+, positive likelihood ratio; LR-, negative likelihood ratio; FC, fold-change; miR, microRNA.
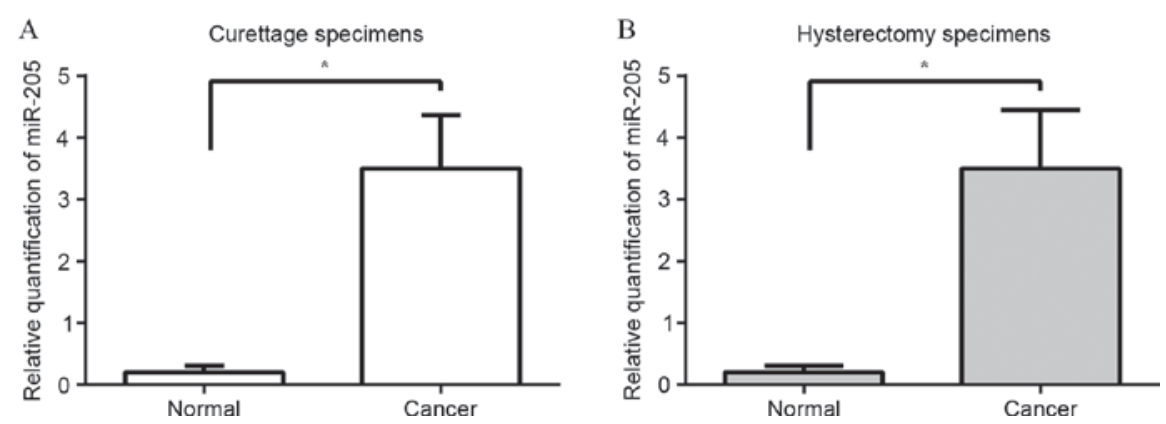

Figure 1. Overexpression of miR-205 in EC. The median expression level of miR-205 was consistently upregulated by 17.5 -fold in thecurettage and hysterectomy specimens of EC compared with their normal counterparts. (A) miR-205 was significantly elevated in the curettage samples of EC. (B) miR-205 was significantly increased in the hysterectomy samples of EC. Statistical analysis was performed by Mann-Whitney U test. "P<0.0001. miR, microRNA; EC, endometrial cancer.

and Fig. 4, patients with a relatively high expression level of miR-205 yielded worse survival times than patients with a low level of miR-205 in the curettage $(\mathrm{P}=0.006)$ and hysterectomy $(\mathrm{P}=0.008)$ groups, respectively. These findings indicated that 
Table IV. Expression of miR-205 (cut-off of 14.5 FC) and histological parameters in curettage specimens predict lymph node metastasis of endometrial cancer patients with clinical stage I disease.

\begin{tabular}{|c|c|c|c|c|c|c|c|c|c|c|c|}
\hline \multirow[b]{2}{*}{ Variable } & \multirow[b]{2}{*}{$\mathrm{n}$} & \multicolumn{3}{|c|}{ Univariate } & \multicolumn{3}{|c|}{ Multivariate } & \multirow[b]{2}{*}{ SNS } & \multirow[b]{2}{*}{ SP } & \multirow[b]{2}{*}{$\mathrm{LR}+$} & \multirow[b]{2}{*}{ LR- } \\
\hline & & OR & $95 \% \mathrm{CI}$ & P-value & OR & $95 \% \mathrm{CI}$ & P-value & & & & \\
\hline Curettage histology & & & & 0.002 & & & 0.02 & 0.45 & 0.88 & 3.75 & 0.62 \\
\hline Low-risk & 228 & 1.00 & & & 1.00 & & & & & & \\
\hline High-risk & 42 & 3.03 & $1.81-4.78$ & & 2.67 & $1.17-3.64$ & & & & & \\
\hline miR-205 expression & & & & 0.001 & & & 0.01 & 0.73 & 0.84 & 4.56 & 0.32 \\
\hline miR-205<14.5 FC & 211 & 1.00 & & & 1.00 & & & & & & \\
\hline $\mathrm{miR}-205>14.5 \mathrm{FC}$ & 59 & 3.12 & $1.97-5.01$ & & 2.09 & $1.05-4.74$ & & & & & \\
\hline Curettage histology+ miR-205 & & & & $<0.001$ & & & 0.01 & 0.83 & 0.84 & 5.19 & 0.20 \\
\hline Low-risk+miR-205<14.5 FC & 207 & 1.00 & & & 1.00 & & & & & & \\
\hline High-risk or/and miR-205>14.5 FC & 63 & 3.07 & $1.08-4.96$ & & 2.47 & $1.12-3.89$ & & & & & \\
\hline
\end{tabular}

Curettage histology low-risk: Endometrioid and grade 1-2; and high-risk: Non-endometrioid or endometrioid grade 3. OR, odds ratios; CI, confidence interval; SNS, sensitivity; SP, specificity; LR+, positive likelihood ratio; LR-, negative likelihood ratio; FC, fold-change; miR, microRNA.

Table V. Multivariate survival analyses for all patients with endometrial cancer.

\begin{tabular}{|c|c|c|c|c|}
\hline Variable & $\mathrm{n}$ & Adjusted HR & $95 \% \mathrm{CI}$ & P-value \\
\hline Age, years & & & & 0.017 \\
\hline$<60$ & 111 & 1.00 & & \\
\hline$\geq 60$ & 249 & 1.14 & $1.05-1.19$ & \\
\hline FIGO stage $^{\mathrm{a}}$ & & & & 0.001 \\
\hline I and II & 306 & 1.00 & & \\
\hline III and IV & 54 & 4.32 & $1.77-7.84$ & \\
\hline Histological grade & & & & 0.003 \\
\hline 1 and 2 & 310 & 1.00 & & \\
\hline 3 & 50 & 3.02 & $1.56-5.04$ & \\
\hline Lymph node metastasis & & & & 0.002 \\
\hline Negative & 314 & 1.00 & & \\
\hline Positive & 46 & 2.97 & $1.14-3.89$ & \\
\hline miR-205 curettage & & & & 0.006 \\
\hline miR-205<16.0 FC & 266 & 1.00 & & \\
\hline miR-205>16.0 FC & 94 & 2.23 & $1.08-3.54$ & \\
\hline miR-205 hysterectomy & & & & 0.008 \\
\hline miR-205<16.0 FC & 268 & 1.00 & & \\
\hline miR-205>16.0 FC & 92 & 2.16 & $1.01-3.23$ & \\
\hline
\end{tabular}

${ }^{\mathrm{a} C r i t e r i a ~ o f ~ t h e ~} 1988$ FIGO staging system. HR, hazard ratio; CI, confidence interval; FC, fold-change; FIGO, international federation of gynecology and obstetrics.

the increased expression of miR-205 in tumor tissues could identify EC patients with unfavorable survival times.

\section{Discussion}

The individualization of lymphadenectomy remains challenging in the surgical management of EC, particularly in clinical stage I disease where lymph node excision has been suggested to provide a survival benefit for high-risk patients, whereas the omission of lymphadenectomy can be adopted in the low-risk group $(31,32)$. Owing to the relatively low incidence of lymph node metastasis in low-risk EC patients, randomized surgical trials with high efficacy are difficult to implement unless an adequate population is available. By 


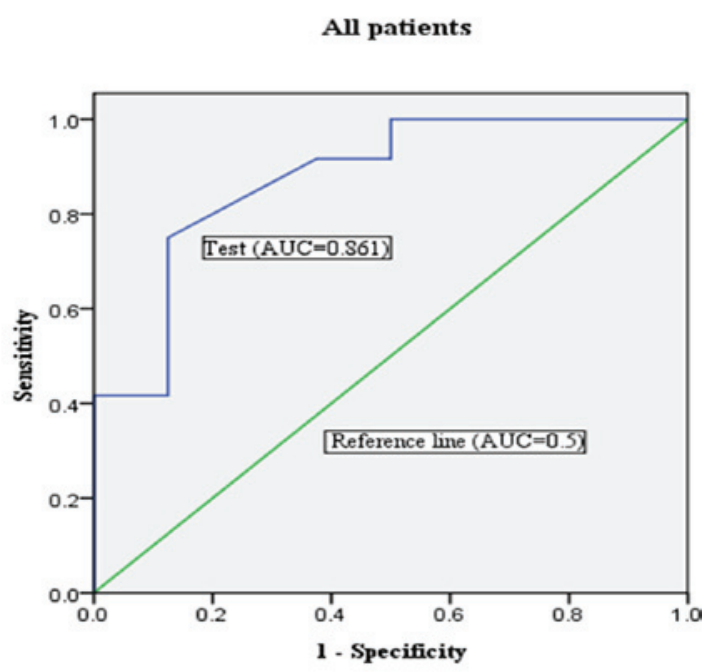

Figure 2. Overexpressed miR-205 in curettage specimens predicts lymph node metastasis for all patients with EC. The cut-off wasa 16.0 fold-change (area under curve $=0.861 ; \mathrm{P}=0.003 ; 95 \% \mathrm{CI}, 0.709-1.000$ ).

\section{Patients with clinical stage I disease}

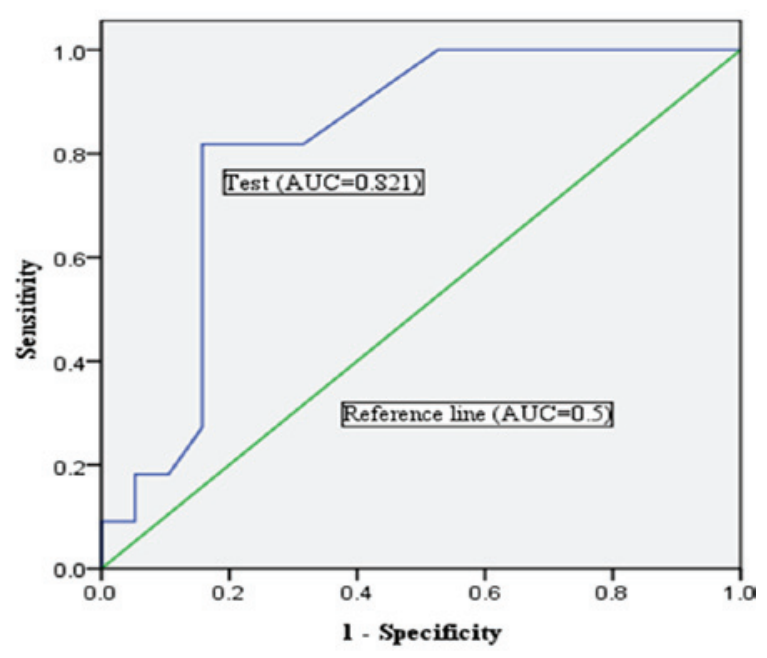

Figure 3. Overexpressed miR-205 in curettage specimens predicts lymph node metastasis for EC patients with clinical stage I disease. The cut-off was a fold-change of 14.5 (AUC=0.821, $\mathrm{P}=0.004,95 \%$ CI 0.666-0.975). AUC, area under receiver operating characteristic (ROC) curve.

contrast, the assessment of novel tools holding potential for identifying patients with a high risk for lymph node involvement is regarded more reasonable, as this pattern would decrease the required sample size in a clinical trial of lymphadenectomy, meanwhile avoiding unnecessary complications from sampling low-risk patients (33).

The pre-operative evaluation of lymph node metastasis represents a crucial step to decide the extent of surgery in EC. Endometrial biopsy is considered to be the cornerstone of the diagnosis and primary surgical treatment planning for EC (34). It has previously been reported that pre-operative histological subtype and grade determined through
$\mathbf{A}$

Curettage specimens

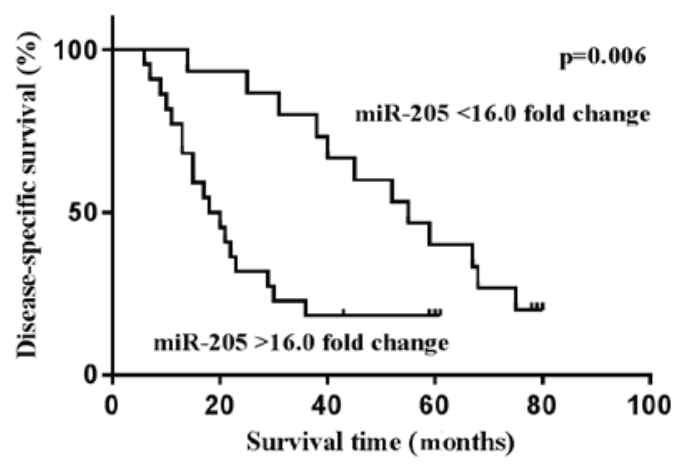

B

Hysterectomy specimens

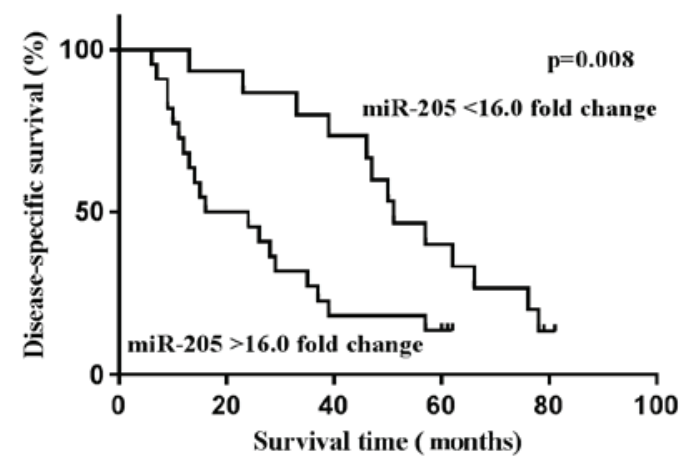

Figure 4. Upregulated expression of miR-205 indicatesa poor prognosis in patients with EC. (A) Patients with a fold-change in miR-205 expression $>16.0$ experienced worse survivalthan patients with a $<16.0$ fold-change in the curettage specimens $(\mathrm{P}=0.006)$. (B) Expression of miR-205 at $>16.0$ fold-change in the hysterectomy specimens was also associated with an unfavorable prognosis in patients with $\mathrm{EC}(\mathrm{P}=0.008)$. Comparison of survival rate between different groups was made by Log-rank test.

endometrial biopsy-derived samples are independent predictors for lymph node metastasis in EC $(35,36)$. However, the predictive efficacy of these two basic histological parameters is not robust enough and remains to be improved.

Of the miRNAs studied that are associated with EC, miR-205 is one of those that is consistently overexpressed. The upregulation of miR-205 was previously reported to be significantly correlated with advanced-stage disease, the occurrence of relapse and poor survival rates in EC $(37,38,30)$. It was revealed that the reduced expression of miR-205 was useful in predicting lymph node involvement in triple-negative breast cancer patients (39), but this result was based on the final pathological reports, which could only be obtained when surgery had been performed.

As pre-operative endometrial biopsy-based detection is a minimally invasive and cost-effective approach, and in view of the stability of miRNAs in FFPE tissues, miR-205 expression was examined in the curettage and hysterectomy specimens of patients with EC in the present study. A good concordance was found concerning the expression of miR-205 between pre-operative and post-operative detection. In line with previous studies, it was demonstrated that the overexpression of miR-205 in the curettage and excised uterus specimens was inversely correlated with disease-specific survival in EC. Moreover, in the current pilot study, the predictive value of miR-205 was first investigated by detecting its expression 
in curettage specimens to identify EC patients at high risk for lymph node metastasis, thus trying to allocate only patients who could potentially benefit from lymphadenectomy to undergo this surgical procedure. The results revealed that lymph node metastasis could be identified by the overexpression of miR-205 for all EC patients, with a sensitivity of $71.4 \%$, a specificity of $80.2 \%$, a positive likelihood ratio of 3.55 , a negative likelihood ratio of 0.36 and an accuracy of $79.4 \%$. Based on a combination of pre-operative histological characteristics and miR-205 expression, the predictive ability of lymph node metastasis could be enhanced to a sensitivity of $80.4 \%$, a specificity of $80.3 \%$, a positive likelihood ratio of 4.00, a negative likelihood ratio of 0.25 and an accuracy of $80.3 \%$.

In particular, the present study focused on the patients with clinical stage I disease, where the role of systemic lymph node excision is not yet well defined. It was found that for clinical stage I disease, lymph node metastasis could be predicted by the overexpression of miR-205, with a sensitivity of $72.5 \%$, a specificity of $84.2 \%$, a positive likelihood ratio of 4.56, a negative likelihood ratio of 0.32 and an accuracy of $83.0 \%$. When combining pre-operative histological characteristics with miR-205 expression, the predictive value of lymph node metastases could be improved to a sensitivity of $82.7 \%$, a specificity of $83.8 \%$, a positive likelihood ratio of 5.19, a negative likelihood ratio of 0.20 and an accuracy of $83.7 \%$. These findings demonstrated that overexpressed miR-205 in curettage specimens could provide additional and useful information for reinforcing risk stratification prior to surgery. On the other hand, the enhanced sensitivity and positive likelihood ratio are beneficial for identifying patients at high risk for lymph node metastasis.

There are also certain limitations to the present study. Although the probability of isolated para-aortic nodal involvement in patients without pelvic nodal metastasis was only $\sim 1 \%$ (40), the fact that only a small proportion of the patients were subjected to pelvic and para-aortic node excision may have affected the results. Furthermore, other factors that can identify high-risk patients, such as myometrial invasion, were not studied, as pre-operative magnetic resonance imaging (MRI) data were available for only a limited number of patients due to the relatively high cost. In fact, pre-operative MRI also has its limitations for the judgment of myometrial invasion, in other words, interobserver variability or inconsistency. Additionally, the present study was limited as it was unicentric. However, we consider that the findings obtained are promising for further studies.

In summary, the present results suggested again that the overexpression of miR-205 was inversely correlated with patient survival. More importantly, the present study has demonstrated, for the first time, that the increased expression of miR-205 in curettage specimens could predict lymph node metastasis with high accuracy in EC, particularly in clinical stage I disease. Moreover, when combined with conventional histological parameters, overexpressed miR-205 could provide additional and useful information, and therefore improve the risk assessment prior to surgical treatment. Further studies with multicenter large cohorts are required to fully validate the potential of miR-205 as a biomarker for the prediction of lymph node metastasis in EC patients.

\section{Acknowledgements}

This study was supported by Ningxia Natural Science Foundation (grant no. NZ14129) and was also partly funded by the National Natural Science Foundation of China (grant no. 81372808), the Science and Technology Development Planning Project of Jinan (grant no. 201303035) and the Technology Development Planning Project of Shandong (grant nos. 2012G0021823 and 2011GSF12122).

\section{References}

1. Siegel R, Naishadham D and Jemal A: Cancer statistics, 2013. CA Cancer J Clin 63: 11-30, 2013.

2. Wong YF, Cheung TH, Lo KW, Yim SF, Siu NS, Chan SC, Ho TW, Wong KW, Yu MY, Wang VW, et al: Identification of molecular markers and signaling pathway in endometrial cancer in Hong KongChinese women by genome-wide gene expression profiling. Oncogene 26: 1971-1982, 2007.

3. Zhang Y, Liu Z, Yu X, Zhang X, Lü S, Chen X and Lü B: The association between metabolic abnormality and endometrial cancer: A large case-control study inChina. Gynecol Oncol 117: 41-46, 2010.

4. Morrow CP, Bundy BN, Kurman RJ, Creasman WT, Heller P, Homesley HD and Graham JE: Relationship between surgical-pathological risk factors and outcome in clinical stage I and II carcinoma of the endometrium: A Gynecologic oncology Group study. Gynecol Oncol 40: 55-65, 1991.

5. Abeler VM and Kjørstad KE: Endometrial adenocarcinoma in Norway. A study of a total population. Cancer 67: 3093-3103, 1991.

6. Kim HS and Song YS: International Federation of Gynecology and Obstetrics (FIGO) staging system revised: What should be considered critically for gynecologic cancer? J Gynecol Oncol 20: 135-136, 2009.

7. ASTEC study group, Kitchener H, Swart AM, Qian Q, Amos C and Parmar MK: Efficacy of systematic pelvic lymphadenectomy in endometrial cancer (MRC ASTEC trial): A randomized study. Lancet 373: 125-136, 2009.

8. Benedetti Panici P, Basile S, Maneschi F, Alberto Lissoni A, Signorelli M, Scambia G, Angioli R, Tateo S, Mangili G, et al: Systematic pelvic lymphadenectomy vs. No lymphadenectomy in early-stage endometrial carcinoma: Randomized clinical trial. J Natl Cancer Lnst 100: 1707-1716, 2008.

9. Chan JK, Cheung MK, Huh WK, Osann K, Husain A, Teng NN and Kapp DS: Therapeutic role of lymph node resection in endometrioid corpus cancer: A study of 12,333 patients. Cancer 107: 1823-1830, 2006

10. Trovik J, Wik E, Stefansson IM, Marcickiewicz J, Tingulstad S, Staff AC, Njolstad TS; MoMaTec Study Group, Vandenput I, Amant F, et al: Stathmin overexpression identifies high-risk patients and lymph node metastasis in endometrial cancer. Clin Cancer Res 17: 3368-3377, 2011.

11. Feng G, Wang X, Cao X, Shen L and Zhu J: ZEB1 expression in endometrial biopsy predicts lymph node metastases in patient with endometrial cancer. Dis Markers 2014: 680361, 2014.

12. Jiang T, Huang L and Zhang S: Preoperative serum CA125: A useful marker for surgical management of endometrial cancer. BMC Cancer 15: 396, 2015.

13. Bansal N, Yendluri V and Wenham RM: The molecular biology of endometrial cancers and the implications for pathogenesis, classification, and targeted therapies. Cancer Control 16: 8-13, 2009.

14. Zhang B, Wang Q and Pan X: MicroRNAs and their regulatory roles inanimals and plants. J Cell Physiol 210: 279-289, 2007.

15. Zhao G, Cai C, Yang T, Qiu X, Liao B, Li W, Ji Z, Zhao J, Zhao H, Guo M, et al: MicroRNA-221 induces cell survival and cisplatin resistance through PI3K/Akt pathway in human osteosarcoma. PLoS One 8: e53906, 2013.

16. Go H, Jang JY, Kim PJ, Kim YG, Nam SJ, Paik JH, Kim TM, Heo DS, Kim CW and Jeon YK: MicroRNA-21 plays an oncogenic role by targeting FOXO1 and activating the PI3K/AKT pathway in diffuse large B-cell lymphoma. Oncotarget 6: 15035-15049, 2015.

17. Li Y, VandenBoom TG II, Kong D, Wang Z, Ali S, Philip PA and Sarkar FH: Up-regulation of miR-200 and let-7 by natural agents leads to the reversal of epithelial-to-mesenchymal transition in gemcitabine-resistant pancreatic cancer cells. Cancer Res 69: 6704-6712, 2009. 
18. Peng Y, Liu YM, Li LC, Wang LL and Wu XL: microRNA-503 inhibits gastric cancer cell growth and epithelial-to-mesenchymal transition. Oncol Lett 7: 1233-1238, 2014.

19. Lu J, Getz G, Miska EA, Alvarez-Saavedra E, Lamb J, Peck D, Sweet-Cordero A, Ebert BL, Mak RH, Ferrando AA, et al: MicroRNA expression profiles classify human cancers. Nature 435: 834-838, 2005.

20. Volinia S, Calin GA, Liu CG, Ambs S, Cimmino A, Petrocca F, Visone R, Iorio M, Roldo C, Ferracin M, et al: A microRNA expression signature of human solid tumors defines cancer gene targets. Proc Natl Acad Sci USA 103: 2257-2261, 2006.

21. Lee TS, Jeon HW, Kim YB, Kim YA, Kim MA and Kang SB: Aberrant microRNA expression in endometrial carcinoma using formalin-fixed paraffin-embedded (FFPE) tissues. PLoS One 8: e81421, 2013.

22. Iorio MV, Visone R, Di Leva G, Donati V, Petrocca F, Casalini P, Taccioli C, Volinia S, Liu CG, Alder H, et al: MicroRNA signatures in human ovarian cancer. Cancer Res 67: 8699-8707, 2007.

23. Qu C, Liang Z, Huang J, Zhao R, Su C, Wang S, Wang X, Zhang R, Lee MH and Yang H: miR-205 determines the radioresistance of human nasopharyngeal carcinoma by directly targeting PTEN. Cell Cycle 11: 785-796, 2012.

24. Gandellini P, Folini M, Longoni N, Pennati M, Binda M, Colecchia M, Salvioni R, Supino R, Moretti R, Limonta P, et al: miR-205 Exerts tumor-suppressive functions in human prostate through down-regulation of protein kinase Cepsilon. Cancer Res 69: 2287-2295, 2009.

25. Boren T, Xiong Y, Hakam A, Wenham R, Apte S, Wei Z, Kamath S, Chen DT, Dressman H and Lancaster JM: MicroRNAs and their target messenger RNAs associated with endometrial carcinogenesis. Gynecol Oncol 110: 206-215, 2008

26. Hiroki E, Akahira J, Suzuki F, Nagase S, Ito K, Suzuki T, Sasano H and Yaegashi N: Changes in microRNA expression levels correlate with clinicopathological features and prognoses in endometrial serous adenocarcinomas. Cancer Sci 101: 241-249, 2010.

27. Campbell K, Nuss RC and Benrubi GI: An evaluation of the clinical staging of endometrial cancer. J Reprod Med 33: 8-10, 1988.

28. Creasman WT, Morrow CP, Bundy BN, Homesley HD, Graham JE and Heller PB: Surgical pathologic spread patterns of endometrial cancer. A gynecologic oncology group study. Cancer 60: (8 Suppl) 2035-2041, 1987.

29. Livak KJ and Schmittgen TD: Analysis of relative gene expression data using real-time quantitative PCR and the 2(-Delta Delta C(T)) Method. Methods 25: 402-408, 2001.

30. Karaayvaz M, Zhang C, Liang S, Shroyer KR and Ju J: Prognostic significance of miR-205 in endometrial cancer. PLoS One 7: e35158, 2012.
31. Todo Y, Kato H, Kaneuchi M, Watari H, Takeda $M$ and Sakuragi N: Survival effect of para-aortic lymphadenectomy in endometrial cancer (SEPAL study): A retrospective cohort analysis. Lancet 375: 1165-1172, 2010.

32. Mitamura T, Watari H, Todo Y, Kato T, Konno Y, Hosaka M and Sakuragi N: Lymphadenectomy can be omitted for low-risk endometrial cancer based on preoperative assessments. J Gynecol Oncol 25: 301-305, 2014.

33. Trovik J, Wik E, Werner HM, Krakstad C, Helland H, Vandenput I, Njolstad TS, Stefansson IM, Marcickiewicz J, Tingulstad S, et al: Hormone receptor loss in endometrial carcinoma curettage predicts lymph node metastasis and poor outcome in prospective multicentre trial. Eur J Cancer 49: 3431-3441, 2013.

34. Dinkelspiel HE, Wright JD, Lewin SN and Herzog TJ: Contemporary clinical management of endometrial cancer. Obstet Gynecol Int 2013: 583891, 2013.

35. Todo Y, Sakuragi N, Nishida R, Yamada T, Ebina Y, Yamamoto R and Fujimoto S: Combined use of magnetic resonance imaging, CA 125 assay, histologic type, and histologic grade in the prediction of lymph node metastasis in endometrial carcinoma. Am J Obstet Gynecol 188: 1265-1272, 2003.

36. Todo Y, Okamoto K, Hayashi M, Minobe S, Nomura E, Hareyama H, Takeda M, Ebina Y, Watari H and Sakuragi N: A validation study of a scoring system to estimate the risk of lymph node metastasis for patients with endometrial cancer for tailoring the indication of lymphadenectomy. Gynecol Oncol 104: 623-628, 2007.

37. Chung TK, Cheung TH, Huen NY, Wong KW, Lo KW, Yim SF, Siu NS, Wong YM, Tsang PT, Pang MW, et al: Dysregulated microRNAs and their predicted targets associated with endometrioid endometrial adenocarcinoma in Hong Kong women. Int J Cancer 124: 1358-1365, 2009.

38. Torres A, Torres K, Pesci A, Ceccaroni M, Paszkowski T, Cassandrini P, Zamboni G and Maciejewski R: Diagnostic and prognostic significance of miRNA signatures in tissues and plasma of endometrioid endometrial carcinoma patients. Int J Cancer 132: 1633-1645, 2013

39. Berber U, Yilmaz I, Narli G, Haholu A, Kucukodaci Z and Demirel D: miR-205 and miR-200c: Predictive micro RNAs for lymph node metastasis in triple negative breast cancer. J Breast Cancer 17: 143-148, 2014.

40. Abu-Rustum NR, Gomez JD, Alektiar KM, Soslow RA, Hensley ML, Leitao MM JR, Gardner GJ, Sonoda Y, Chi DS and Barakat RR: The incidence of isolated paraaortic nodal metastasis in surgically staged endometrial cancer patients with negative pelvic lymph nodes. Gynecol Oncol 115: 236-238, 2009. 